\title{
Health vigilance concerning Acinetobacter baumannii bacteremia at the mohammed VI university hospital of oujda (morocco): epidemiological profile and antibiotic resistance
}

\author{
Loubna Yacoubi ${ }^{1,}{ }^{*}$, Soumia Farih $^{1}$, Noussaiba Benhamza ${ }^{1}$ Abderazzak Seddari ${ }^{1}$, and Adil maleb ${ }^{1}$ \\ ${ }^{1}$ Microbiology Laboratory. Mohammed VI University Hospital of Oujda (Morocco), Medicine and Pharmacy of Oujda, Mohammed I \\ University, Morocco.
}

\begin{abstract}
The objective of this work is to determine the epidemiological profile of Acinetobacter $b$ aumannii (A.baumannii ) bacteremia in the microbiology laboratory of CHU Mohammed VI of Oujda and its antibiotic resistance rates. This is a retrospective and descriptive study of 27 months fromJune 24, 2016to September 19, 2018 including all positive blood cultures processed in the microbiology laboratory in accordance with REMIC (reference in medical microbiology)and EUCAST(European Committee on Antimicrobial Susceptibility Testing). Contaminated blood cultures were excluded. As results we collected 863 positive blood cultures, A. baumannii accounted for 7.41\% $(n=64) .67 \%(n=43)$ of the strains were isolated from patients hospitalized in intensive care (adults, children and newborns). The two main risk factors described in patients with our series were wearing of intravascular device in $55 \%(n=35)$ Immunosuppression in $22 \% n=14)$. A. baumannii bacteremia was associated with care in $37.5 \%(n=24) .75 \% \quad(n=48)$ of A. baumannii isolates were resistant to carbapenems. No strain of A. baumannii was resistant to colistin. In light of these results strengthening the control and prevention measures for healthcare associated infections would be the most reliable way to limit the spread of A. baumannii in our establishment.
\end{abstract}

\section{Introduction}

A baumannii is a Gram-negative, non -motile, strictly aerobic, non-fermentative, non-sporing, coccobacillus. The ability of A. baumannii to form biofilms contributes to its survival in adverse environmental conditions including hospital environments and medical devices[1].

Of particular importance is the ability of Acinetobacter to cause bacteremia, especially in critically ill patients, the clinical course of which may range from a benign transient bacteremia to fulminant septic shock [2].

The existence of multi-resistant strains places A. baumannii among the organisms that threaten the current therapeutic management and therefore the choice of molecules during probabilistic treatment is a real challenge. Carbapenems have long been considered as the treatment of choice for Acinetobacter infections. Today, this class is threatened by the emergence of resistance, favored by its increasing use in connection with the emergence of multi-resistant enterobacteria. [3].
Patients with multi-drug resistant A. baumannii nosocomial bacteremia have longer lengths of stay, higher costs, and higher mortality rates [4].

The objective of this work is to determine the epidemiological profile of A. baumannii bacteremia and its antibiotic resistance rates.

\section{Materials and methods}

This was a retrospective and descriptive study from June 24, 2016 to September 19, 2018 and included all positive blood cultures processed in the microbiology laboratory ; Contaminated blood cultures were excluded.

The samples are taken by venipuncture and then injected into two vials: one aerobic and one anaerobic, in our study the blood culture vials used are BD BACTEC ${ }^{\text {TM }}$ vials (Becton Dickinson) . after verification of non-compliance; The vials are recorded and incubated in the BD FX 400 automaton for a maximum of 7 days

*Loubna Yacoubi : loubnayacoubi1993@gmail.com 
(beyond this time, the microorganisms detected are usually contaminants).

The vials detected positive by the BD BACTEC are discharged from the automaton and analyzed. The culture was performed on enriched and selective agar media. Incubation was performed at $37^{\circ} \mathrm{C}$ for 24 to 48 hours.

The identification of A. baumannii at the laboratory level was based on conventional morphological, cultural, biochemical and antigenic characteristics. Biochemical identification was done by Api galleries, determined with the BD Phoenix 100.

Antibiotic susceptibility testing was performed in accordance with the recommendations of the Antibiotic susceptibility Committee of the French Society of Microbiology (CA-SFM) and the EUropean Committee on Antimicrobial Susceptibility Testing (EUCAST) [5].

The results were interpreted in accordance with the recommendations of the medical microbiology reference system (REMIC) [6] .

\section{Results:}

\section{Epidemiology}

During the study period, we collected 863 positive blood cultures, with A. baumannii accounting for $7.41 \%$ $(n=64)$. The majority of A. baumannii strains were isolated from newborns, which represented $47 \%$ of cases $(\mathrm{n}=30) .19$ patients were female $(30 \%)$ versus 45 male $(70 \%)$, representing a sex ratio of 2.36 .

$67 \%(n=43)$ of the strains were isolated from patients hospitalized in the intensive care unit (adults, children and neonates), by medical departments with $22 \%(n=14)$ of the isolates, and surgical departments with $5 \%(n=3)$ of the isolates, the hospital department was not specified for $6 \%(n=4)$ of the patients.

The two main risk factors described in the patients of our series were: Intravascular device wear in $55 \%(n=35)$, Immunosuppression in $22 \% \mathrm{n}=14$ ).

Bacteremia caused by A. baumannii were associated with care in $37.5 \%(\mathrm{n}=24)$.

\section{Resistance profile}

Of the 64 strains of A. baumannii isolated in the microbiology laboratory, $87 \%$ were resistant to Ciprofloxacin and Gentamicin, $86 \%$ to Cefepime and Levofloxacin, $85 \%$ to Ceftazidime, Ticarcillin, and Ticarcillin/clavulanic acid combination, 75\% $(n=48)$ of A. baumannii isolates were resistant to carbapenems .No A. baumannii strain was resistant to colistin. ( Fig 1)

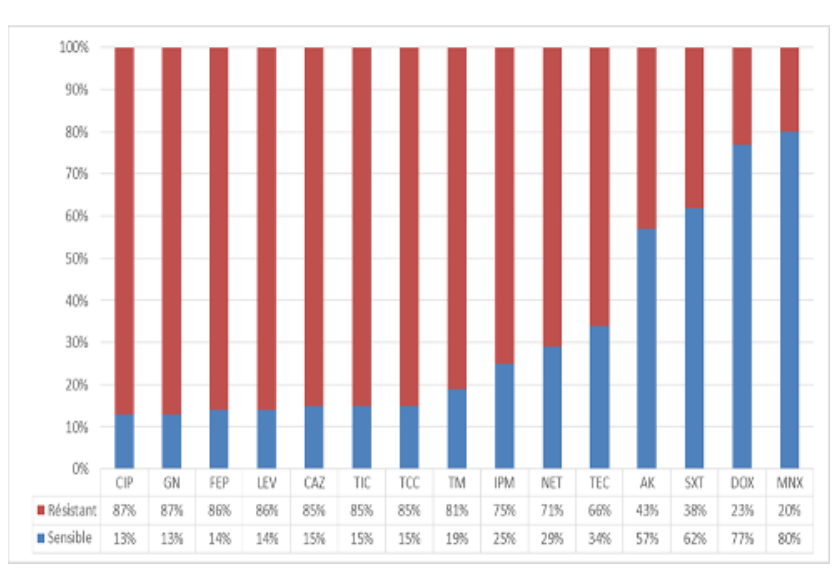

Fig 1. Resistance rates of Acinetobacter Baumannii.

Ciprofloxacin (CIP), Gentamicin (GN), Cefepime (FEP), Levofloxacin (LEV), Ceftazidime (CAZ), Ticarcillin (TIC), Ticarcillin/clavulanic acid (TCC), Tobramycin (TM), Imipenem (IPM), Netilmicin (NET), Tetracycline (TEC), Amikacin (AK), Trimethoprim sulfamethoxazole (SXT), Doxycyline (DOX), Minocycline (MNX).

\section{Discussion}

\section{Epidemiology}

During the period of our study, A. baumannii represented $5.8 \%(n=64)$ of all positive blood cultures. This figure remains lower than that reported in the literature. The different studies [7-10] underline that A. baumannii is the most frequent non-fermenting Gram-negative bacillus incriminated in bacteremia.

Being an opportunistic pathogen, Acinetobacter baumannii often affects fragile patients such as the immunocompromised, low birth weight neonates, and intensive care patients $[11,12]$.

In our study, the average age was 25 years, which is due to the fact that about $47 \%$ of $\mathrm{A}$. baumannii bacteremias were isolated in newborns. This average age is far from the data of the literature, which note a predominance in adult subjects with an average age of 67 years $[2,13,14]$.

In the present study, we also noted a male predominance with a sex ratio of 2.36 , which is in line with the data in the literature. This male predominance is reported by several studies $[2,13,14]$.

The male predominance may be explained by the finding that A. baumannii bacteremia is often associated with underlying conditions such as smoking, alcoholism, diabetes and other chronic lung diseases [15].

\footnotetext{
*Loubna Yacoubi : loubnayacoubi1993@gmail.com
} 
In our study, a large proportion $(67 \%)$ of $\mathrm{A}$. baumannii bacteremias originated in the intensive care unit. These results are consistent with the literature, as the intensive care unit is the most common site for A. baumannii infections, but with varying rates. The rate found in this study is lower than that reported in Italy in $2019(85 \%)$ [16] , but remains higher than that reported in Greece in 2016 (53.9\%)[17].

We pointing out that only $22 \%(n=14)$ of patients were immunocompromised while $55 \%(\mathrm{n}=35)$ of patients wore an intravascular device and. These rates are lower than those reported in Lebanon in 2017[2] and in China in 2016[18 ].

\section{Resistance profile}

According to a recent WHO report, A. baumannii becomes a major threat to public health when it acquires resistance to broad-spectrum antibiotics, such as carbapenems [19]. Current findings suggest that the battle against this pathogen is being lost [20] due to the limited alternatives for treating the infections it causes [21] and the lack of public health interventions to control antibiotic resistance.

From 2011 to 2014, 2337 A. baumannii were collected from 453 hospital sites in 48 countries as part of the SMART continuous surveillance initiative. Rates of multidrug-resistant acinetobacter baumannii were lowest in North America (47\%) and highest in Europe and the Middle East (>93\%),[22]. In our study we noted multiresistance of A. baumannii to the tested antibiotics.

The resistance to ciprofloxacin in the different studies is higher than $50 \%$ which is in line with the data of our study [23-28].

Resistance to aminoglycosides was $87 \%$ for gentamycin and $81 \%$ for tobramycin, these rates are higher than those reported in Casablanca [28] and Tunisia [27].

Resistance to amikacin is variable: $43 \%$ in our study against $66 \%$ in Casablanca [28], 79\% in Tunisia [27], and $82 \%$ in Iran [23].

Our strains are less resistant to imipenem (75\% in our study compared to $93 \%$ in China, $96.9 \%$ in Iran and $88.6 \%$ in Tunisia).

For the combination of trimethoprim and sulfamethoxazole $38 \%$ of our strains were resistant, this rate remains far from that reported in Casablanca (77\%), Tunisia (51.5\%) and China (93.4\%)

Colistin is the last resort for the treatment of multiresistant Acinetobacter baumannii. Unfortunately,

\footnotetext{
*Loubna Yacoubi : loubnayacoubi1993@gmail.com
}

resistance to colistin has been reported worldwide. The highest rate of resistance has been reported in Asia, followed by Europe [29]. in our study no A. baumannii strain was resistant to colistin.

\section{Conclusion}

Our study showed the important place that occupies the bacteremia with A. baumannii within our hospital structure in particular in intensive care unit, in fragile patients carriers of invasive materials or presenting a ground of immunodepression, indeed this bacteria became a not negligible therapeutic headache considering the emergence of the multi resistant strains to the antibiotics.

In light of these results, strengthening healthcareassociated infection control and prevention measures would be the most reliable way to limit the spread of A. baumannii in our institution.

\section{References}

1. Eze EC, Chenia HY, El Zowalaty ME. Acinetobacter baumannii biofilms: effects of physicochemical factors, virulence, antibiotic resistance determinants, gene regulation, and future antimicrobial treatments. Infect Drug Resist. ( 2018 ) ;11:2277-2299.

2. Ballouz, Tala, et al. "Risk Factors, Clinical Presentation, and Outcome of Acinetobacter Baumannii Bacteremia." Frontiers in Cellular and Infection Microbiology, vol. 7, ( 2017 ),

3. Valérie Delbos. Manifestations cliniques et traitement des infections à Acinetobacter baumannii, Revue Francophone des Laboratoires, Volume 2012 , Issue 441, (April 2012), Pages 59-65

4. Aguirre-Avalos G, Mijangos-Méndez JC, AmayaTapia G. Bacteriemia por Acinetobacter baumannii. Rev Med Inst Mex Seguro Soc. (2010 ) ;48 (6) :625-634.

5. Recommandations du CASFM / EUCAST ( 2015 ) v.1.0. Comité de l'Antibiogramme de la Société Française de Microbiologie (SFM) - European Committee on Antimicrobial Susceptibility Testing (EUCAST).

6. Société Française de Microbiologie . Bactériémies et fongémies - hémocultures. Rémic : Référentiel en microbiologie( 2015) :137-152 p

7. Kant Khanal, Laxmi. "Bacteriological Profile of Blood Culture and Antibiogram of the Bacterial Isolates in a Tertiary Care Hospital." International Journal of Health Sciences and Research, vol. 10, no. 8, ( 2020$)$

8. Bandy A, Almaeen AH (2020) Pathogenic spectrum of blood stream infections and resistance pattern in 
Gram-negative bacteria from Aljouf region of Saudi Arabia. PLoS ONE 15(6): e0233704.

9. Ali Z.Le .profil bactérilogique des bactériémies et l'état de résistance aux antibiotiques Thèse de Médecine $N^{\circ} 219$ ( 2019 ). Faculté de Médecine et de Pharmacie de Marrakech

10. Ahmed L.Aspects microbiologiques et résistance aux antibiotiques de bactéries isolées d'hémoculture à l'hôpital militaire Moulay Ismail de Meknès de 2015 à 2018 . Thèse de Médecine $\mathrm{N}^{\circ} 078$ (2020). Faculté de Médecine et de Pharmacie de Fès.

11. Gramatniece, A., et al. "Control of Acinetobacter Baumannii Outbreak in the Neonatal Intensive Care Unit in Latvia: Whole-Genome Sequencing Powered Investigation and Closure of the Ward." Antimicrobial Resistance \& Infection Control, vol. 8, no. 1, ( 2019 )

12. Tang, C.q., et al. "Epidemiology and Outcomes of Bloodstream Infections in 177 Severe Burn Patients from an Industrial Disaster: a Multicentre Retrospective Study." Clinical Microbiology and Infection, vol. 24, no. 2, ( 2018 ).

13. Yang, Shuangshuang, et al. "Determinants of Mortality in Patients with Nosocomial Acinetobacter Baumannii Bacteremia in Southwest China: A Five-Year Case-Control Study." Canadian Journal of Infectious Diseases and Medical Microbiology, vol. 2018, ( 2018 ) , pp. 1-9.

14. Papathanakos, Georgios, et al. "Colistin-Resistant Acinetobacter Baumannii Bacteremia: A Serious Threat for Critically Ill Patients." Microorganisms, vol. 8, no. 2, ( 2020 ) , p. 287.

15. Villar M, Cano ME, Gato E, Garnacho-Montero J, Miguel Cisneros J, Ruíz de Alegría C, et al. Epidemiologic and clinical impact of Acinetobacter baumannii colonization and infection: a reappraisal. Medicine (Baltimore). (2014 );93[5]:202-10.

16. Russo, Alessandro, et al. "Bloodstream Infections Caused by Carbapenem-Resistant Acinetobacter Baumannii: Clinical Features, Therapy and Outcome from a Multicenter Study." Journal of Infection, vol. 79, no. 2, (2019), pp. 130-138.,

17. Maraki, Sofia, et al. "A 5-Year Surveillance Study on Antimicrobial Resistance OfAcinetobacter BaumanniiClinical Isolates from a Tertiary Greek Hospital." Infection \& Chemotherapy, vol. 48, no. 3, ( 2016 ) , p. 190.,.,

18. L, Qiao, et al. "Analysis of Risk Factors on Prognosis of Acinetobacter Baumannii Bloodstream Infection." Clinical Proteomics and Bioinformatics, vol. 1, no. 2, ( 2016 ),

19. World Health Organization . WHO publishes list of bacteria for which new antibiotics are urgently needed . (2017) .

20. Protic D, Pejovic A, Andjelkovic D, Djukanovic N, Savic D, Piperac P, et al. Nosocomial infections caused by acinetobacter baumannii: Are we losing

\footnotetext{
*Loubna Yacoubi : loubnayacoubi1993@gmail.com
}

the battle? Surg Infect (Larchmt)(2016) ;17(2):236-242.

21. Isler B, Yohei D, Bonomo RA, Paterson D. New Treatment Options against Carbapenem-Resistant Acinetobacter baumannii Infections. Antimicrob Agents Chemother. (2019);63(1):1-17.

22. Lob, Sibylle $\mathrm{H}$ et al. "Regional differences and trends in antimicrobial susceptibility of Acinetobacter baumannii." International journal of antimicrobial agents vol. 47,4 (2016): 317-323.

23. Afhami, Shirin, et al. "Antimicrobial Resistance Pattern of Acinetobacter; a Multicenter Study, Comparing European Committee on Antimicrobial Susceptibility Testing (EUCAST) and the Clinical and Laboratory Standards Institute (CLSI); Evaluation of Susceptibility Testing Methods for Polymyxin." Immunopathologia Persa, vol. 7, no. $1,(2020)$.

24. Zuniga-Moya JC, Caballero CA, Loucel-Linares M, Benitez MJ, Zambrano-Garcia E, Fajardo LV, et al. Antimicrobial profile of Acinetobacter baumannii at a tertiary hospital in Honduras: a cross-sectional analysis. Rev Panam Salud Publica.(2020);44:e46.

25. Shi, Jingyi, et al. "Multidrug Resistant and Extensively Drug Resistant Acinetobacter Baumannii Hospital Infection Associated with High Mortality: a Retrospective Study in the Pediatric Intensive Care Unit.” BMC Infectious Diseases, vol. 20, no. 1, ( 2020),

26. Konca C, Tekin M, Geyik M. Susceptibility Patterns of Multidrug-Resistant Acinetobacter baumannii. Indian J Pediatr. 2021 Feb;88(2):120-126

27. Jaidane, Nadia \& Mansour, Wejdene \& Bonnin, Rémy \& Ghardallou, Mariem \& Chaouch, Cherifa \& Rachida, Golli \& Nesrine, Kalboussi \& Noureddine, Boujaafar \& Olfa, Bouallegue \& Naas, Thierry. (2019). Temporal Variation in Antibiotic Resistance of Acinetobacter baumannii in a Teaching Hospital in Tunisia: Correlation with Antimicrobial Consumption. The Open Microbiology Journal. 13. 106-111.

28. El Kettani, Assiya et al. "Prevalence of Acinetobacter baumannii bacteremia in intensive care units of Ibn Rochd University Hospital, Casablanca." Iranian journal of microbiology vol. 9 (6) (2017): 318-323.

29. Yun Cai, Dong Chai, Rui Wang, Beibei Liang, Nan Bai, Colistin resistance of Acinetobacter baumannii: clinical reports, mechanisms and antimicrobial strategies, Journal of Antimicrobial Chemotherapy, Volume 67, Issue 7, ( July 2012 ) , Pages 16071615 , 